\title{
THE SUMMABILITY OF FOURIER SERIES
}

BY M. H. STONE

In an article of mine which appeared recently in the Transactions of this Society, I applied the general theorems deduced to the particular case of Fourier series. In these applications I omitted an essential step.* The object of this note is to extend some of the methods of that paper in the case of Fourier series, to prove some general theorems about the summability of infinite series, and to supply thereby the reasoning which I omitted in the earlier treatment.

M. Riesz has given a general method of summing an infinite series, $\dagger$ of which a special case may be described as follows: a series of functions of the real variable $x$,

$$
u_{0}(x)+u_{1}(x)+u_{2}(x)+\cdots,
$$

is said to be summable $\left(\alpha^{\Delta}, \delta\right)$ to the value $U(x)$ if

$$
S_{\Omega}^{\Delta, 8}(x) \rightarrow U(x), \quad \Omega \rightarrow+\infty,
$$

where

$$
S_{\Omega}^{\Delta, \delta} \equiv \sum_{\alpha=0}^{<\omega}\left(1-\frac{\alpha^{\Delta}}{\Omega}\right)^{\delta} u_{\alpha}(x), \Omega=\omega^{\Delta}, \Delta>0, \delta \geqq 0 .
$$

Here $\omega$ is a continuous variable. A more general method of summation is obtained if $\omega$ is restricted to a sequence of values $0<\omega_{1}<\omega_{2}<\cdots, \omega_{\nu} \rightarrow+\infty$. If

* Stone, Transactions of this Society, vol. 28 (1926), pp. 695-761. See Theorems 14, 33, 35, 36, 37, 38.

$\dagger$ M. Riesz, Comptes Rendus, vol. 149 (1909), pp. 909-912; Comptes Rendus, vol. 152 (1911), pp. 1651-1654; Proceedings of the London Society, (2), vol. 22 (1923-24), pp. 412-419; Hardy and Riesz, The General Theory of Dirichlet's Series (Cambridge Tracts, No. 18, 1915), Chapters IV and V; Hardy, Proceedings of the London Society, (2), vol. 15 (1915-16), pp. 7288. 


$$
S_{\Omega}^{\Delta, \delta}(x) \rightarrow U(x),
$$

the series may be referred to as summable $\left(\alpha^{\Delta}, \delta ; \omega_{\nu}\right)$ to the value $U(x) .{ }^{*}$ Clearly summability by the first method implies summability by the second; but the converse is not true in general. $\dagger$ The omission referred to above is due to the neglect of this fact. In order to remedy it, we may follow one of two courses: we may extend the method involving summability $\left(\alpha^{\Delta}, \delta ; \omega_{\nu}\right)$ which was employed in that paper to yield a method involving summability $\left(\alpha^{\Delta}, \delta\right)$; or we may prove that with respect to Fourier series the two methods are interchangeable. We shall investigate both possibilities.

It is convenient to introduce the definition of a restricted kind of equivalence.

Definition. Two definitions of summability are said to be equivalent with respect to a class of series when every series of that class summable by one method is summable by the other to the same sum; and when, further, uniform summability on a range of the variable $x$ in one case implies uniform summability on the same range in the other.

In examining the two types of summability defined above and their equivalence with respect to the class of Fourier series and certain related classes of series, we shall restrict $\Delta$ to integral values. It is first necessary to find a compact analytical expression for the sums $S_{\Omega}^{n}, \delta$. Without loss of generality we may suppose that $\delta$ is greater than zero; and may assume that the Lebesgue-integrable function $f(x)$

* This type of summability has been applied to the study of Fourier series by W. H. Young, Leipziger Berichte, vol. 63 (1911), pp. 369-387; by Stone, loc. cit.; and by Bailey, Annals of Mathematics, vol. 27 (1926), pp. 69-102.

$\dagger$ Riesz, Comptes Rendus, vol. 152 (1911), pp. 1651-1654; Proceedings of the London Society, vol. 22 (1923-24), pp. 412-419. Bailey, loc. cit., Lemmas III and IV, states two equivalence theorems which in view of Riesz's London paper do not seem to be correct; his reference to Young seems to be in error, as Young, loc. cit., does not give any discussion of equivalence. 
whose Fourier series is under consideration satisfies the condition $\int_{0}^{1} f d y=0$, since this restriction modifies only the constant term of the Fourier series on $(0,1)$. If we let $G(x, y ; \lambda)$ be the Green's function for the differential system $u^{\prime}+\lambda u=0, u(0)-u(1)=0$, so that*

$G(x, y ; \lambda)=\left\{e^{\lambda(y-x)} ; 0\right\}+e^{\lambda(y-1)-\lambda x} /\left(1-e^{-\lambda}\right), \quad R(\lambda) \geqq 0$, $G(x, y ; \lambda)=\left\{0 ;-e^{\lambda(y-x)}\right\}+e^{\lambda y+\lambda(1-x)} /\left(1-e^{\lambda}\right), R(\lambda) \leqq 0$,

and if we choose appropriate contours $S_{1}$ and $S_{2}$ enclosing the poles of $\int_{0}^{1} f(y) G(x, y ; \lambda) d y$ on the upper and lower half of the $\lambda$-plane respectively, we may write

$$
\begin{array}{rlrl}
S_{\Omega}^{n, \delta} & =\frac{1}{2 \pi i} \int_{S_{1}} \int_{0}^{1} f(y)\left[1-(-i \lambda / \Lambda)^{n}\right]^{\delta}\left\{\frac{\partial^{k} G}{\partial x^{k}} ; \frac{\partial^{k} G}{\partial x^{k}}\right\} d y d \lambda \\
& +\frac{1}{2 \pi i} \int_{S_{2}} \int_{0}^{1} f(y)\left[1-(+i \lambda / \Lambda)^{n}\right]^{\delta}\left\{\frac{\partial^{k} G}{\partial x^{k}} ; \frac{\partial^{k} G}{\partial x^{k}}\right\} d y d \lambda, \\
\Lambda & =2 \pi \omega, & (k=0,1,2, \cdots),
\end{array}
$$

for the sum obtained from the $k$ th derived series of the Fourier series on the interval $(0,1)$ for the function $f(x) . \dagger$ The contours $S_{1}$ and $S_{2}$ which we shall employ may be described as the boundaries of the two semicircular regions into which the circle $|\lambda| \leqq \Lambda$ is divided by the axis of reals. It will be convenient to denote the circular arcs in $S_{1}$ and $S_{2}$ by $C_{1}$ and $C_{2}$ respectively. The formula given above can then be verified by the calculus of residues; it is valid even when $C_{1}$ and $C_{2}$ pass through poles of the Green's function, because of the presence of the factors $\left(1-( \pm i \lambda / \Lambda)^{n}\right)^{\delta}$, $\delta>0$.

We first study the contribution to $S_{\Omega}^{n, \delta}$ from the bracketed terms in the Green's function. The method followed is sufficiently exemplified by the treatment of the portion of this contribution arising from that part of $S_{1}$ for which

* Stone, loc. cit., p. 717

† Stone, loc. cit., $\$ 2$ and Lemma 13. 
$R(\lambda) \geqq 0$. The integral over this path may be replaced by an integral from 0 to $\Lambda i$ along the axis of imaginaries, and therefore becomes

$$
\begin{aligned}
& \frac{1}{2 \pi i} \int_{0}^{\Lambda i} \int_{0}^{x} f(y)\left[1-(-i \lambda / \Lambda)^{n}\right]^{\delta}(-\lambda) e^{\lambda(y-x)} d y d \lambda \\
& =\frac{1}{2 \pi} \int_{0}^{x} \int_{0}^{1} f(-z+x)\left(1-\phi^{n}\right)^{\delta}(-i \Lambda \phi)^{k} \Lambda e^{-\Lambda i \phi z} d \phi d z
\end{aligned}
$$

by the change of variables $z=x-y, \phi=-\lambda i / \Lambda$. The total contribution from the bracket terms is found in this way to be

$$
\begin{gathered}
\frac{1}{2 \pi} \int_{0}^{1-x} f(z+x) \Lambda^{k+1} \Phi_{n, k, \delta}(\Lambda z) d z \\
+\frac{(-1)^{k}}{2 \pi} \int_{0}^{x} f(-z+x) \Lambda^{k+1} \Phi_{n, k, \delta}(\Lambda z) d z,
\end{gathered}
$$

where

$$
\Phi_{n, k, \delta}(\alpha)=\int_{0}^{1}\left(1-\phi^{n}\right)^{\delta} \phi^{k}\left[(-i)^{k} e^{\alpha i \phi}+i^{k} e^{-\alpha i \phi}\right] d \phi .
$$

By integrating $k+1$ times by parts in the integral defining $\Phi_{n}, k, \delta(\alpha)$ we can obtain a more convenient form for this function when $\delta \geqq k$. We denote by $h_{m}(\phi)$ the $m$ th derivative of the function $\left(1-\phi^{n}\right)^{\delta} \phi^{k}$. If $\delta$ is greater than or equal to $k$ the functions $h_{0}, \cdots, h_{k}$ are continuous functions of $\phi$ on the interval $(0,1)$; and $h_{k+1}$ is continuous except at $\phi=1$ and is integrable in the sense of Lebesgue on $(0,1)$. Furthermore, the equations $h_{m}(0)=h_{m}(1)=0$ are satisfied when $m=0, \cdots, k-1$; and $h_{k}(1)=0$ if $\delta>k$. Thus the result of integrating $k+1$ times by parts is seen to be*

* In a similar integration by parts which occurs in the proof of Theorem 35 of my paper cited above one term has been overlooked; the necessary correction is easily supplied. 


$$
\begin{aligned}
\Phi_{n, k, \delta}(\alpha) & =(-1)^{k} \alpha^{-k} \int_{0}^{1} h_{k}(\phi)\left[(-1)^{k} e^{\alpha i \phi}\right. \\
& \left.+(-1)^{k} e^{-\alpha i \phi}\right] d \phi \\
& =2 \alpha^{-k} \int_{0}^{1} h_{k}(\phi) \cos \alpha \phi d \phi \\
& =2 \alpha^{-k-1} h_{k}(1) \sin \alpha-2 \alpha^{-k-1} \int_{0}^{1} h_{k+1}(\phi) \sin \alpha \phi d \phi
\end{aligned}
$$

By the theorem of Riemann-Lebesgue, the integral in the last expression is $o(1)$.

We now apply this result to the further simplification of the contribution from the bracket terms investigated above. We restrict the variable $x$ to lie on a fixed interval $(a, b)$ completely interior to $(0,1)$. We can then choose a positive number $A$ less than both $a$ and $1-b$. Now

$$
\begin{aligned}
& \mid \frac{1}{2 \pi} \int_{A}^{1-x} f(z+x) \Lambda^{k+1} \Phi_{n, k, \delta}(\Lambda z) d z \\
& \quad-\frac{h_{k}(1)}{\pi} \int_{A}^{1-x} f(z+x) z^{-k-1} \sin \Lambda z d z \mid
\end{aligned}
$$

is less than or equal to

$$
\begin{array}{r}
\quad \frac{1}{\pi} \int_{A}^{1-x}|f(z+x)| z^{-k-1}\left|\int_{0}^{1} h_{k+1}(\phi) \sin \Lambda z \phi d \phi\right| d z \\
\leqq A^{-k-1} \eta \int_{A}^{1-x}|f(z+x)| d z \leqq A^{-k-1} \eta \int_{0}^{1}|f(y)| d y \leqq \epsilon
\end{array}
$$

for $\Lambda z \geqq \Lambda A_{-}^{-}$sufficiently large. We can treat the second part of the contribution in the same way. Thus we can write the whole expression as

$$
\begin{aligned}
\frac{1}{2 \pi} \int_{0}^{A} & {\left[f(z+x)+(-1)^{k} f(-z+x)\right] \Lambda^{k+1} \Phi_{n, k, \delta}(\Lambda z) d z } \\
& +\frac{h_{k}(1)}{\pi}\left[\int_{A}^{1-x} f(z+x) z^{-k-1} \sin \Lambda z d z\right. \\
& \left.+\int_{A}^{x}(-1)^{k} f(-z+x) z^{-k-1} \sin \Lambda z d z\right]+o(1) .
\end{aligned}
$$


If we change the variables of integration in the second term, it may be written

$$
\frac{h_{k}(1)}{\pi} \int_{0}^{1} f(y) K(x, y ; \Lambda) d y
$$

where

$$
\begin{aligned}
& K(x, y ; \Lambda)=\sin \Lambda(y-x) /(y-x)^{k+1}, \quad 0 \leqq y \leqq x-A, \\
& K(x, y ; \Lambda)=0 \quad x-A<y<x+A, \\
& K(x, y ; \Lambda)=\sin \Lambda(y-x) /(y-x)^{k+1}, \quad x+A \leqq y \leqq 1,
\end{aligned}
$$

for $a \leqq x \leqq b$; and this integral can easily be shown to be $o(1)$ on $(a, b)$ by the theory of singular integrals.* The final form of the contribution from the bracket terms in $G$ is therefore

$$
\begin{gathered}
\frac{1}{2 \pi} \int_{0}^{A}\left[f(z+x)+(-1)^{k} f(-z+x)\right] \Lambda^{k+1} \Phi_{n, k, \delta}(\Lambda z) d z \\
+o(1)
\end{gathered}
$$

uniformly on $(a, b)$, when $\delta$ is a positive number greater than or equal to $k$.

We now turn to the study of the contribution to $S_{\Omega}{ }^{n, \delta}$ from the remainder of the Green's function, under the assumption that $x$ lies on $(a, b)$ and that $\delta$ is a positive number greater than or equal to $k$. First we show that the integrals taken along the real axis are negligible, and then discuss the integrals over $C_{1}$ and $C_{2}$.

The contribution from the positive real axis is seen to be

$$
\begin{gathered}
\frac{1}{2 \pi i} \int_{0}^{\Lambda}\left\{\left[1-(-i \lambda / \Lambda)^{n}\right]^{\delta}-\left[1-(i \lambda / \Lambda)^{n}\right]^{\delta}\right\} \\
\times \int_{0}^{1}\left[f(y)(-\lambda)^{k} e^{\lambda(y-1)-\lambda x} /\left(1-e^{-\lambda}\right)\right] d y d \lambda .
\end{gathered}
$$

Under our assumption that $\int_{0}^{1} f d y$ vanishes the integrand is analytic in $\lambda$ at the origin. Furthermore

* Lebesgue, Annales de Toulouse, (3), vol. 1 (1909), pp. 52-55. 
1927.]

$$
\begin{array}{r}
\int_{0}^{1}\left[f(y)(-\lambda)^{k} e^{\lambda(y-1)-\lambda x} /\left(1-e^{-\lambda}\right)\right] d y \\
=O\left(\lambda^{k} e^{-\lambda x}\right)=O\left(\lambda^{k} e^{-\lambda a}\right) .
\end{array}
$$

If we split the integral over $(0, \Lambda)$ into two others, one over $\left(0, \Lambda^{1 / 2}\right)$, the other over $\left(\Lambda^{1 / 2}, \Lambda\right)$, we find the first to be

$$
O\left(\int_{0}^{\Lambda^{1 / 2}} O\left(\Lambda^{-n / 2}\right) O\left(\lambda^{k} e^{-\lambda a}\right) d \lambda\right)=o(1)
$$

and the second to be

$$
O\left(\int_{\Lambda^{1 / 2}}^{\Lambda} O(1) O\left(\lambda^{k} e^{-\lambda a}\right) d \lambda\right)=o(1) .
$$

In exactly the same way we can show that the integral over the negative axis of reals is also $o(1)$ uniformly on $(a, b)$.

In discussing the integrals over the $\operatorname{arcs} C_{1}$ and $C_{2}$ we shall give the details of the method used only for the integral taken over the arc $c$ of $C_{1}$ lying on the first quadrant. This may be written

$$
\int_{0}^{1} f(y) k(x, y ; \Lambda) d y
$$

where

$$
k(x, y ; \Lambda)=\frac{1}{2 \pi i} \int_{c}^{\left[1-(-i \lambda / \Lambda)^{n}\right]^{\delta}(-\lambda)^{k} e^{\lambda(y-1)-\lambda x}} d \lambda .
$$

It is our purpose to show that

$$
\begin{aligned}
k(x, y ; \Lambda) & =O(1), \\
\int_{\alpha}^{\beta} k(x, y ; \Lambda) d y & =o(1), \quad 0 \leqq \alpha<\beta \leqq 1,
\end{aligned}
$$

uniformly on $(a, b)$; the theory of singular integrals then shows that $\int_{0}^{1} f(y) k(x, y ; \Lambda) d y$ is $o(1)$ uniformly on $(a, b) .^{*}$ We introduce the variable $\theta$ defined by the equation $e^{-i \theta}$ $=-i \lambda / \Lambda$. When $\lambda$ is on $c, \theta$ is a real variable on the interval $(0, \pi / 2)$, so that

\footnotetext{
* Lebesgue, loc, cit.
} 


$$
\begin{aligned}
{\left[1-(-i \lambda / \Lambda)^{n}\right] \delta } & =\theta^{\delta} O(1), \\
(-\lambda)^{k} e^{\lambda(y-1)-\lambda x} & =O\left(\Lambda^{k} e^{-\Lambda A \theta / 2}\right), \\
\int_{\alpha}^{\beta}(-\lambda)^{k} e^{\lambda(y-1)-\lambda x} d y & =O\left(\Lambda^{k-1} e^{-\Lambda A \theta / 2}\right), \\
d \lambda & =\Lambda O(1) d \theta .
\end{aligned}
$$

For a given value of $\Lambda$, we determine an integer $l$ such that $(2 l-1) \pi \leqq \Lambda \leqq(2 l+1) \pi$. If we then set $\lambda=\mu+2 l \pi i$ we find

$$
1 /\left(1-e^{-\lambda}\right)=1 /\left(1-e^{-\mu}\right)=O(1+1 /|\mu|)
$$

in the region composed of the half-plane $R(\mu) \geqq m>0$ and the rectangle $0 \leqq \mathbb{R}(\mu) \leqq m,-3 \pi / 2 \leqq J(\mu) \leqq+3 \pi / 2$. If $\Lambda$ and $l$ are large $c$ lies on the corresponding region for the variable $\lambda$. In terms of the variable $\theta$ we have

$$
\begin{aligned}
1 /|\mu|=(1 / \Lambda)\left|e^{-i \theta}-2 l \pi / \Lambda\right|=(1 / \Lambda)[ & 4 l \pi(1-\cos \theta) / \Lambda \\
& \left.+(1-2 l \pi / \Lambda)^{2}\right]^{1 / 2} \\
= & O\left\{(1 / \Lambda)[4 l \pi(1-\cos \theta) / \Lambda]^{1 / 2}\right\}=O\{1 /(\Lambda \theta)\},
\end{aligned}
$$

when $\lambda$ is on $c$. Consequently

$$
1 /\left(1-e^{-\lambda}\right)=O\{1 /(\Lambda \theta)\}+O(1) .
$$

Thus

$$
\begin{aligned}
k(x, y ; \Lambda)= & O\left(\int_{0}^{\pi / 2} \Lambda^{k} \theta^{\delta-1} e^{-\Lambda A \theta / 2} d \theta\right) \\
& +O\left(\int_{0}^{\pi / 2} \Lambda^{k+1} \theta^{\delta} e^{-\Lambda A \theta / 2} d \theta\right) \\
= & O(1),
\end{aligned}
$$

if $\delta$ is a positive number not less than $k$; and

$$
\begin{aligned}
\int_{\alpha}^{\beta} k(x, y ; \Lambda) d y= & O\left(\int_{0}^{\pi / 2} \Lambda^{k-1} \theta^{\delta-1} e^{-\Lambda A \theta / 2} d \theta\right) \\
& +O\left(\int_{0}^{\pi / 2} \Lambda^{k} \theta^{\delta} e^{-A \Lambda \theta / 2} d \theta\right) \\
= & O(1 / \Lambda)
\end{aligned}
$$


uniformly on $(a, b)$ under the same conditions.* This establishes the desired result. It is evident now that the integrals over the other parts of $C_{1}$ and $C_{2}$ will yield to similar treatment. Thus we can state the first theorem.

THEOREM I. The sum $S_{\Omega}^{n, \delta}$ formed for the kth derived series of the Fourier series for a Lebesgue-integrable function $f(x)$ on the interval $(0,1)$ can be expressed as

$S_{\Omega}^{n, \delta}=\frac{1}{2 \pi} \int_{0}^{A}\left[f(z+x)+(-1)^{k} f(-z+x)\right] \Lambda^{k+1}$
$\cdot \Phi_{\delta, k, n}(\Lambda z) d z+o(1)$,

uniformly $0<a \leqq x \leqq b<1$, where $\delta$ is a positive number not less than $k$ and $A$ is a positive number less than both $a$ and $1-b$. Here $\Lambda=2 \pi \omega$ is a continuous variable.

This theorem amounts to an extension of the methods of our paper cited above to include a treatment of summability $\left(\alpha^{n}, \delta\right)$; it serves to bridge the gap which was left in the proofs of those theorems enumerated in the first footnote. By using this theorem we can arrive at further theorems, concerning the relation of summability $\left(\alpha^{n}, \delta\right)$ to summability $\left(\alpha^{n}, \delta ; \omega_{\nu}\right)$ with respect to the class of Fourier series and the class of derived series of Fourier series.

If $f(x)$ is absolutely continuous - that is, if it is an integral in the sense of Lebesgue-we can integrate by parts in the integral appearing in Theorem I. In view of the fact that

$$
\int \Phi_{n, k, \delta}(\Lambda z) d z=-\Phi_{n, k-1, \delta}(\Lambda z) / \Lambda, \quad k \geqq 1,
$$

we obtain for $k \geqq 1$

$$
\begin{aligned}
& S_{\Omega}^{n, \delta}=-\Lambda^{k}\left[f(A+x)+(-1)^{k} f(-A+x)\right] \Phi_{n, k-1, \delta}(\Lambda A) \\
& +\frac{1}{2 \pi} \int_{0}^{A}\left[f^{\prime}(z+x)+(-1)^{k-1} f(-z+x)\right] \Lambda^{k} \Phi_{n, k-1, \delta}(\Lambda z) d z \\
& +o(1) .
\end{aligned}
$$

* See Stone, loc. cit., Lemma V, for a more detailed discussion of the final steps here. 
To evaluate the first term here we treat $\Phi_{n, k-1,8}(\Lambda A)$ by the method of integration by parts outlined above. Since $\delta \geqq k$, we have

$$
\Phi_{n, k-1, \delta}(\alpha)=-2 \alpha^{-k} \int_{0}^{1} \frac{d^{k}}{d \phi^{k}}\left[\left(1-\phi^{n}\right)^{\delta} \phi^{k-1}\right] \sin \alpha \phi d \phi .
$$

In consequence the first term is $o(1)$ uniformly $a \leqq x \leqq b$. By repeating this process we obtain the second theorem.

THEOREM II. If $f^{(l-1)}(x)$ is absolutely continuous,

$$
\begin{aligned}
S_{\Omega}^{n, 8}= & \frac{1}{2 \pi} \int_{0}^{A}\left[f^{(l)}(z+x)+(-1)^{(k-l)} f^{(l)}(-z+x)\right] \Lambda^{k-l+1} \\
& \cdot \Phi_{n, k-l, \delta}(\Lambda z) d z+o(1),
\end{aligned}
$$

uniformly on $(a, b)$, if $\delta \geqq k \geqq 1$ and $l \leqq k$. In other words, the sum formed for the kth derived series for $f(x)$ and the sum formed for the $(k-l)$ th derived series for $f^{(l)}(x)$ have a difference which is uniformly $o(1)$ on $(a, b), l=0, \cdots, k$.

We now compare summability $\left(\alpha^{n}, \delta\right)$ and $\left(\alpha^{n}, \delta ; \omega_{\nu}\right)$ for a Fourier series, $\delta>0$, under the hypothesis that $\omega_{\nu+1}-\omega_{\nu}$ $=o(1)$. A necessary and sufficient condition that the series be summable $\left(\alpha^{n}, \delta ; \omega_{\nu}\right)$ to the sum $S(x)$ is that

$$
\begin{aligned}
I_{\nu}=\frac{1}{2 \pi} \int_{0}^{A} F(z) \Lambda_{\nu} \Phi_{n, 0, \delta}\left(\Lambda_{\nu} z\right) d z=o(1) \\
\quad F(z)=f(z+x)+f(-z+x)-2 S(x), \Lambda_{\nu}=2 \pi \omega_{\nu},
\end{aligned}
$$

the limit being approached uniformly on any closed set $\varepsilon$ on which the summability is uniform.* We consider the quantity

$$
J_{\nu}=\frac{1}{2 \pi} \int_{0}^{A} F(z)\left[\Lambda \Phi_{n, 0, \delta}(\Lambda z)-\Lambda_{\nu} \Phi_{n, 0, \delta}\left(\Lambda_{\nu} z\right)\right] d z
$$

* For amplification of the reasoning here, see Stone, loc. cit., Theorem 14. 
where $\Lambda_{\nu} \leqq \Lambda \leqq \Lambda_{\nu+1}$. By the process of integration by parts used above

$$
\begin{aligned}
& \Lambda \Phi_{n, 0, \delta}(\Lambda z)-\Lambda_{\nu} \Phi_{n, 0, \delta}\left(\Lambda_{\nu} z\right)= \\
& \int_{0}^{1}\left\{\frac{d}{d \phi}\left(1-\phi^{n}\right)^{\delta} \cdot\left[\sin \Lambda_{\nu} \phi z-\sin \Lambda \phi z\right] / z\right\} d z=O(1),
\end{aligned}
$$

since

$$
\left(\sin \Lambda_{\nu} \phi z-\sin \Lambda \phi z\right) / z=\phi \cos \Lambda_{\nu}^{\prime} \phi z\left(\Lambda_{\nu}-\Lambda\right),
$$

$\Lambda_{\nu}<\Lambda_{\nu}^{\prime}<\Lambda$ by the law of the mean. Consequently,

$$
\begin{aligned}
\left|J_{\nu}\right| \leqq & K \int_{0}^{A}|F(z)| d z \leqq K\left(\int_{0}^{A}|f(z+x)| d z\right. \\
& \left.+\int_{0}^{A}|f(-z+x)| d z+A|S(x)|\right)
\end{aligned}
$$

where $K$ is a suitably chosen positive constant. The last expression is $o(1)$ for each value of $x$ when $A \rightarrow 0$. The first two terms are uniformly $o(1)$ on $(a, b)$ by a fundamental property of the Lebesgue integral. On any closed set $\varepsilon$ on which the given Fourier series is uniformly summable $\left(\alpha^{n}, \delta ; \omega_{\nu}\right)$, the function $S(x)$ is continuous and bounded; on this set $\varepsilon$, therefore, $A|S(x)|$ is uniformly $o(1)$ when $A \rightarrow 0$. Thus by choosing $A$ sufficiently small we can make $\left|J_{\nu}\right|<\epsilon / 2$, where $\epsilon$ is a preassigned positive constant. The value assigned to $A$ may be taken independent of $x$, when $x$ lies on a closed set $\varepsilon$ on which the summability $\left(\alpha^{n}, \delta ; \omega_{\nu}\right)$ is uniform. Now

$$
S_{\Omega}^{n, \delta}-S(x)=I_{\nu}+J_{\nu}+o(1),
$$

where $I_{\nu}=o(1)$, for any value of $x$ at which $S(x)$ exists. When, for such a value of $x$, a value of $A$ has been determined so that $\left|J_{\nu}\right|<\epsilon / 2$, a number $L$ can be found so that for $\Lambda_{\nu+1} \geqq \Lambda \geqq \Lambda_{\nu} \geqq L$ we have

Thus we find

$$
\left|I_{\nu}+o(1)\right|<\epsilon / 2 \text {. }
$$

$$
S_{\Omega}^{n, \delta}-S(x)=o(1),
$$


the limit being uniform on any closed set $\mathcal{E}$ on which the summability $\left(\alpha^{n}, \delta ; \omega_{\nu}\right)$ is uniform. Thus we can assert that summability $\left(\alpha^{n}, \delta ; \omega_{\nu}\right)$ and summability $\left(\alpha^{n}, \delta\right)$ are equivalent on $(a, b)$ with respect to the class of Fourier series.

In order to extend the result about equivalence to the whole interval $(0,1)$, we need only appeal to an elementary property of Fourier series. If $f(x)$ is defined outside the interval $(0,1)$ by the periodic relation $f(x+1)=f(x)$, it is found that the Fourier series for $f(x)$ and $f(x-1 / 2)$ have a simple and important relationship: when the variable $x$ in the second series is replaced by $x+1 / 2$, the first series results. Thus the behavior of the Fourier series for $f(x)$ at $x=0$ or $x=1$ can be completely discussed by considering the behavior of the Fourier series for $f(x-1 / 2)$ at $x=1 / 2$. It is thus seen that summability $\left(\alpha^{n}, \delta ; \omega_{\nu}\right)$ and summability $\left(\alpha^{n}, \delta\right)$ are equivalent with respect to the class of Fourier series, without restriction as to the range considered.

THEOREM III. If $\omega_{\nu+1}-\omega_{\nu}=o(1)$, then summability $\left(\alpha^{n}, \delta ; \omega_{\nu}\right)$ and summability $\left(\alpha^{n}, \delta\right)$ are equivalent with respect to the class of Fourier series.

By combining Theorems II and III we find without difficulty a more general result.

THEOREM IV. If $\omega_{\nu+1}-\omega_{\nu}=o(1)$, then summability $\left(\alpha^{n}, \delta\right)$ and summability $\left(\alpha^{n}, \delta ; \omega_{\nu}\right)$ are equivalent with respect to the class of kth derived series of the Fourier series of functions whose $(k-1)$ th derivatives are absolutely continuous, $\delta \geqq k \geqq 1$.

Columbia University 